\title{
Crisis Informatics and Collaboration: A Brief Introduction
}

\author{
Volkmar Pipek ${ }^{1}$, Sophia B. Liu ${ }^{2} \&$ Andruid Kerne ${ }^{3}$ \\ ${ }^{1}$ Institute for Information Systems, University of Siegen, Siegen, Germany (E-mail: \\ volkmar.pipek@uni-siegen.de); ${ }^{2}$ U.S. Geological Survey,St. Petersburg Coastal and Marine \\ Science Center, St. Petersburg, FL, USA (E-mail: sophialiu@usgs.gov); ${ }^{3}$ Interface Ecology \\ Lab, Texas A\&M University, College Station, TX, USA (E-mail: andruid@cse.tamu.edu)
}

\section{Crisis Informatics from a CSCW Point of View}

Major crises and disasters, like the September 11th attacks, Hurricane Katrina, and the Sendai earthquake, constitute a ripe domain for CSCW concerns, as they involve collaboration among individuals, organizations and society as a whole. CSCW issues arise across all phases of emergency management, from initial planning and preparedness, through the detection of a crisis event, and into the response, recovery and mitigation phases. In many crisis scenarios, the quality of the collaboration among governmental, professional, volunteer, and citizen responders in crisis management greatly affects the impact on loss of lives and property.

Crisis Informatics takes an interdisciplinary perspective on the socio-technical, informational and collaborative aspects of developing and using technologies and information systems in the context of the full disaster lifecycle-preparedness, warning, impact, response, recovery, and mitigation phases. Crisis Informatics views emergency management as a socio-technical system, in which information is disseminated within and among official and public channels and entities. Research wrestles with methodological concerns as it strives to develop socially- and behaviorally-informed theories, policies, and development of information and communication technology (ICT). As the challenges of crisis management grow more complex from the increase in vulnerability to hazards of all types, the opportunities to collaborate using a rapidly growing set of ICTs and collaborative technologies (e.g., social networking platforms, mobile devices with integrated cameras, location-aware services, multi-touch surfaces, crowdsourcing systems, web-based systems) urge us to find new ways of understanding, conceptualizing and evaluating possible use cases for these collaborative technologies in emergency management and response. 'Collaborative resilience' strategies can help improve collaboration quality, but such strategies and the ICTs being developed and used in crisis situations need to be explored in terms of describing and exchanging best practices, designing appropriate 
coordination mechanisms, and offering technological support for collaboration across organizational boundaries.

While the potentials for collaborative action when facing a crisis have dramatically improved with the emergence of social media and ubiquitous computing, it is less clear what aspects of the crisis domain constitute an interesting and distinguished field for $\mathrm{CSCW}$ research. Crisis management comes with notions of infrastructures and their breakdown, of urgency, of preparedness, and of coping and recovery work. But if we consider other fields, like health informatics, knowledge management, workflow management or industrial production, we would find similar notions already. What may qualify crisis management as a distinguishable research discourse are considerations of scale, speed, (lack of) control, and complexity in organizational practice.

\subsection{CSCW Research Arenas in Crisis Informatics}

For this Special Issue, we invited a broad landscape of possible research arenas:

- User experience studies and design approaches of presenting, visualizing, and interacting with social media data, crowdsourcing systems, crisis mapping applications, virtual communities, networked organizations, etc. that facilitate collaboration in crisis management;

- Studies and human-centered design approaches of inter-organizational coordination mechanisms, technological concepts and prototypes, and participatory development of emergency management technology that support collaboration in crisis situations;

- Critical studies, methodological considerations, and design approaches of collaborative systems that address ethical, legal, and social issues including security and privacy issues in developing, using, and researching emergency management information systems that involve collaborations between governmental, professional, volunteer, and citizen responders in crisis management;

- Theoretical considerations regarding collaborative resilience and emergency response as a socio-technical system, as well as studies of and concepts for establishing and supporting 'collaborative resilience' that involves collaborative services and technologies to improve the resilience of cities, infrastructures, logistic chains, etc. through public-private partnerships.

- Studies and design approaches of serious games and gamification of crisis management applications for emergency response training, raising awareness, and conducting research;

- Case Studies from practitioners explaining uses of collaborative technologies in the field and discussing potential solutions and real-world applications to give insights into best practices and lessons learned;

- Different international perspectives of socio-cultural differences in uses of collaborative ICTs and participatory decision-making in crisis management. 
Not all arenas were addressed by the submitting authors, but we were able to attract a variety of scholars from different disciplines for this Special Issue.

\section{The Contributions in This Special Issue}

The Call for Paper for this Special Issue was answered by 29 author teams, of which 16 chose to submit a full paper based on the feedback they got on the initial abstract they submitted. In two rounds of revisions, supported by about forty reviewers, six papers were selected for this Special Issue that presented thorough analyses and/or innovative concepts of collaboration to understand the complexity of coordination in crisis situations that can better inform the design of future collaborative systems in this application domain. We start with high-level approaches to developing interorganizational coordination mechanisms for crisis response organizations (CROs) - emergency management agencies, non-profit organizations, and spontaneous volunteer networks - and then continue on with papers that have a more narrow focus on the use of specific technology to facilitate collaboration in crisis management.

Benedikt Ley, Thomas Ludwig, Volkmar Pipek, Dave Randall, Christian Reuter and Torben Wiedenhöfer describe in their paper "Information and Expertise Sharing in Inter-Organizational Crisis Management" a long-term study on the development of an inter-organizational socio-technical infrastructure for exchanging information and expertise among different CROs. The combination of a (restricted) social media site with two embedded modules that provided map-based information and facilitated expertise exchange proved to be successful particularly to support practices of improvisation in crisis management. Improvisation work is often necessary to gather all necessary information on the existing crisis situation, and to include the expertise necessary to assess this information when deciding how to prioritize issues and how to proceed with coping and recovery work. The modules of a lightweight inter-organizational tool for shared situation assessment (ISAC), and of a shared information repository (IOIR) that allows users to annotate, discuss and manage the information sources the different CRO bring to the table proved to be helpful Ley et al. derived design requirements for supporting improvisation work that can also inform other solutions.

Sophia B. Liu's paper "Crisis Crowdsourcing Framework: Designing Strategic Configurations of Crowdsourcing for the Emergency Management Domain" provides us with a comprehensive overview of the crowdsourcing phenomena in the context of crises. Her paper presents a series of vignettes illustrating the evolution of crisis crowdsourcing, which spontaneously emerged after the 2010 Haiti earthquake, to more established forms of public engagement during recent crises. Best practices extracted from the vignettes illustrate the formalization of crisis crowdsourcing, resulting in innovative interfaces designed to support the articulation work needed during the spontaneous efforts. This led to the development of a framework that strategically guides the development of a crisis crowdsourcing system.

This Crisis Crowdsourcing Framework is a comprehensive and methodical approach to determining the why, who, what, when, where, and how aspects of a 
crowdsourcing system, as well as the social, technological, organizational, and policy (STOP) interfaces to manage the articulation work involved with reducing the complexity of coordinating across these salient dimensions. The paper offers an example of how the framework was applied to develop a crowdsourcing system called iCoast at the U.S. Geological Survey.

Dharma Dailey and Kate Starbird's paper "Journalists as Crowdsourcerers: Responding to Crisis by Reporting with a Crowd" provides a detailed account of one specific practice of crowdsourcing, and address the risks and necessities of information sharing in crisis situation from a citizen-oriented perspective. The focus of their study are social media, but in connection with traditional media and their protocols and practices. In particular their ethnographic study covers the journalistic practices of information sharing guiding a community of (information) crowdsourcerers to form an ad-hoc mesh network that produces information to help CROs as well as citizens affected in managing the crisis caused by Hurricane Irene. They discuss their results against the existing discourse on 'infrastructuring' that aims to understand and support the emergence of infrastructures.

While Liu as well as Dailey and Starbird describe successful approaches to operationalize social media for crisis response, Andrea Tapia and Kathleen Moore's paper "Good Enough is Good Enough: Overcoming Disaster Response Organizations" Slow Social Media Data Adoption" points towards the reluctance of professional CROs to embrace the new opportunities social media platforms offer. They start with the requirement of CROs to base decisions only on 'trustworthy' data, often categorically excluding data generated by or in social media. This need is contrasted with the fact that in the early stages of crisis response, necessary information is often limited. In their interview study with a range of $\mathrm{CRO}$ officials, they describe how social media are indeed already in use by CROs, but with a focus on the people with whom they are in contact, not with the data that is produced or shared. They show how crisis responders' needs for information and its availability through information are already driving changes in practice, which needs to go further. They argue for a critical perspective of appropriately knowing when and what social media to use in disaster response, while emphasizing that using networks of trusted people, in addition to ad hoc needs, is the basis for validation.

Hemant Purohit, Andrew Hampton, Shreyansh Bhatt, Valerie Shalin, Amit Sheth, and John Flach's paper "Identifying Seekers and Suppliers in Social Media Communities to Support Crisis Coordination" offers an approach to derive trustworthy and useful information from social media particularly from Twitter. With their 'Twitris' system, they aim to identify, extract and process information on actors seeking or providing resources that may be helpful in a crisis. Their concept of combining Natural Language Processing with annotations as metadata for every tweet considered to be relevant, while at the same time bridging the gap between professional CRO with their defined, formal terminologies and the ordinary citizens or volunteers with their more informal language provides a promising way to overcome information overload of CRO in social media. The system has been validated using a Twitter dataset from the 2010 Haiti earthquake. 
Athula Ginige, Luca Paolino, Marco Romano, Monica Sebillo, Genoveffa Tortora, and Giuliana Vitiello's paper "Information Sharing among Disaster Responders - An Interactive Spreadsheet-Based Collaboration Approach" concludes our Special Issue with an approach to developing a crisis management system based on a spreadsheet-mediated collaboration among on-site emergency responders and decision makers. Using a participatory design approach, they developed a mobile application that allows CRO to gather and share information organized through spreadsheets. The concept has been evaluated based on a set of earthquake management scenarios for high-risk areas in Italy.

\section{Emerging Collaboration Themes in Crisis Informatics}

The papers in this special issue as well as the other manuscripts that were submitted primarily showed how new forms of collaborative work and new conceptualizations of coordination and collaboration in a networked world are needed for the field of Crisis Informatics to explain crisis response as a form of groupwork. From a CSCW perspective, we would raise three types of issues to consider in future work.

\subsection{Organizations and Division of Work}

Although represented by regional entities, we find a society-wide delegation scheme of coping and recovery work, with several large crisis response organizations (CROs), such as emergency management agencies, non-profit organizations, and spontaneous volunteer networks, that develop and specialize on particularly relevant services like search and rescue efforts, restoring critical infrastructure, and maintaining public order at the local, state, and national level. There are defined competencies and responsibilities for every organization through established plans and procedures to warrant a quick but structured reaction; however, situational aspects lead to overlapping decision-making contexts that require inter-organizational coordination. Every organization has established structural and operational characteristics that often combine a hierarchical command structure with distributed teams on site and at regional command centers to better coordinate crisis response efforts in the impact zone. There are also a number of inter-organizational coordination mechanisms, but the resulting division of work is highly situational and thus difficult to anticipate requiring improvisation and pre-negotiated processes and routines.

\subsection{Organizations and Their 'customers'}

Another significant component is the 'customer relation' aspects of emergency services. The 'customers' - the citizens affected by a crisis event-rely and partially depend on emergency services. As a crisis emerges, they often turn from passive customers that need to be taken care of into crisis 
responders acting on their own behalf while engaging in coping and recovery work to help themselves, their neighbors and communities. A surge of volunteerism from citizens not directly affected also emerges during crises, but official actors and particularly professional CROs often treat these volunteers as 'involuntary collaborators' whose competencies and intentions are difficult to assess. Thus, a complex collaboration arena emerges, in which CROs partially act vis-à-vis the citizens affected when active service requests towards CROs significantly increase from critical infrastructure and relief resources breaking down. Partially, CROs act jointly with the citizens visà-vis the circumstances causing the crisis.

On the other hand, there have been notable examples where well-organized volunteer efforts by engaged citizens significantly alleviated the problems caused by the crisis. These coordination efforts and relations between organizations and individuals during crisis response may evolve and sustain themselves into the next crisis. Citizens can be actively involved in activities aiming to improve preparedness of CRO and communities; however, there may be more controversial issues like overlapping responsibilities of CRO or the justification of decisions that resulted in negative consequences for some citizens. These dynamics feed back into the internal organization of CRO, creating the need for detailed documentation of decisions and activities with a tendency to be less transparent for fear of legal consequences. This limits opportunities to create a mutual awareness of activities, decisions and resources to support improvisational practices.

\subsection{The Impact of Hazards Leading to Crises}

A third force to consider is how natural, technological, and social hazards (e.g. hurricanes, nuclear disasters, political unrest) create uncertainty that can significantly impact collaboration and communication infrastructures, which is important to consider when developing concepts and tools for collaborative systems. As a consequence of the uncertainties from crises, another distinctive contingency of Crisis Informatics research are methodological limits to applying standard ethnographic approaches to analytical and design-oriented research. Crises cannot always be anticipated, making systematic research difficult to plan for. Some research practices require certain skills and equipment to document emergent and spontaneous emergency practices (e.g. basic firefighting skills and breathing equipment), and there are crisis situations where it may be a strong disturbance even if researchers remain passive observers. Therefore, CSCW research in Crisis Informatics has to rely on research to understand 'real-world' practices through ethnographic studies of emergency training and exercises. As a research practice that may be the best we can do, but it remains important to remain sensible to the limitations in interpreting the relevance of these materials as 'real' practices. 


\section{Conclusion}

Research in civil security and crisis management usually aims to improve the 'resilience' of a system (e.g., a company, a city, a community, a nation), its ability to resist disturbances of its consistent state as well as its ability to return to this consistent state once being disturbed. It has been a remarkable development that even at important publication venues in crisis management, the analysis of and the design for collaborative settings has become the predominant research discourse (see e.g. the proceedings of the ISCRAM 2014 conference, recent issues of journals like the International Journal on Information Systems in Crisis Response and Management (IJISCRAM) or the Journal on Homeland Security and Emergency Management (JHSEM)). The path to improving resilience in practice may, however, remain difficult, as relying on collaboration always means to risk one's own autonomy. As the development of collaborative systems creates more fluid and efficient ways of organizing the division of labor for responding to, recovering from, and preparing for the next crisis, the challenge is finding good transitions into a world of information and communication technology systems that enable mutual awareness and offer more options for interactions during and between crises for crisis response organizations as well as the citizens affected. We consider the contributions in this Special Issue an important step towards this and hope readers may also find ways to expanding the field of Crisis Informatics.

\section{Acknowledgments}

We would like to thank the Journal editor Kjeld Schmidt for his guidance in producing this special issue, and Jubilyn Hilario and Lester de Leon from the Springer editorial office for their tireless efforts in accompanying the editorial process. Lastly, we would like to thank the reviewers that provided valuable feedback on the manuscripts: Mohammad-Ali Abbasi, Ban Al-Ani, Jørgen P. Bansler, Matthew Bietz, Marcos Borges, Monika Buscher, John M. Carroll, Paloma Diaz, David Diez, Joel Fisher, Daniela Fogli, Sean P. Goggins, Maaike Harbers, Brent Hecht, Amanda Hughes, Murray Jennex, Michael Lindell, Kevin McDougall, Maria Normark, Leysia Palen, Jiri Panek, Konstantia Pantidi, Luca Paolino, Hemant Purohit, David Randall, Christian Reuter, Raz Schwartz, Bryan Semaan, Steven D. Sheetz, Kate Starbird, Andrea H. Tapia, Zach Toups, Sarah Vieweg, and Joanne White. 